FNT/T-95/16

\title{
HYPERON RADIATIVE DECAYS IN THE BOUND STATE SOLITON MODEL
}

\author{
Carlos L. SCHAT ${ }^{a}$, Carlo GOBBI ${ }^{b}$, and Norberto N. SCOCCOLA ${ }^{a, c}$ 更 \\ ${ }^{a}$ Physics Department, Comisión Nacional de Energía Atómica, Av.Libertador 8250, \\ (1429) Buenos Aires, Argentina. \\ ${ }^{b}$ Department of Theoretical and Nuclear Physics, University of Pavia, \\ and INFN, Sezione di Pavia, via Bassi 6, I-27100 Pavia, Italy. \\ c INFN, Sezione di Milano, via Celoria 16, I-20133 Milano, Italy.
}

May 1995

\begin{abstract}
The radiative decays of hyperons are studied in the framework of the bound state soliton model. Detailed predictions for the total decay widths and the E2/M1 ratios corresponding to decuplet-to-octet electromagnetic transitions are presented in relation to future planned experiments at CEBAF and Fermilab. The results are compared to those obtained in quark based models.
\end{abstract}

${ }^{\dagger}$ Fellow of the CONICET, Argentina. 
In the near future, experiments at CEBAF[1] and Fermilab[2] will provide new and precise information about the electromagnetic decays of excited hyperons. Therefore, it is of great interest to investigate these transitions in different theoretical frameworks and compare various model predictions. In fact, transitions between hadron states are more sensitive to their internal structure than hadron spectra, and usually offer a more stringent test of hadron models than "diagonal" properties like masses and magnetic moments. Calculations of hyperon radiative decays have been available

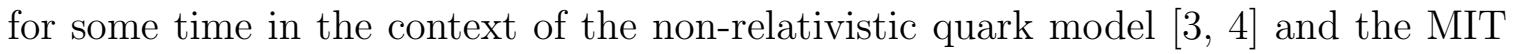
bag model model [4]. More recently this type of transitions has also been examined within heavy baryon chiral perturbation theory [5] and a lattice quenched QCD simulation [6]. The purpose of the present article is to analyze the hyperon radiative decays in the framework of the bound state soliton model [9, [10]. Here, we will concentrate on the decuplet-to-octet electromagnetic transitions. $\Lambda(1405)$ radiative decays as described in this model have been studied in Ref.[11]. For the decupletto-octet decays both M1 and E2 multipole transitions are allowed. Of course, unless they are suppressed by some particular selection rule, the M1 transitions are expected to be strongly dominant over the E2 ones. Nevertheless, the ratio E2/M1 turns out to be a rather important quantity since it reflects the possible existence of charge deformations of the baryon states. In fact, the E2/M1 ratio for the $\Delta \rightarrow N \gamma$ decay has recently received considerable attention both theoretically (see e.g. Refs. [5, 6, 7 and references therein) and experimentally (see e.g. Ref. [8]). For this reason, we will study not only the total decay widths but also the E2/M1 ratios corresponding to the hyperon radiative decays mentioned above.

In the bound state soliton model, one starts with an effective $S U(3)$ chiral action which includes an appropriate symmetry breaking term. We use

$$
\Gamma=\int d^{4} x\left\{-\frac{f_{\pi}^{2}}{4} \operatorname{Tr}\left(L_{\mu} L^{\mu}\right)+\frac{1}{32 e^{2}} \operatorname{Tr}\left[L_{\mu}, L_{\nu}\right]^{2}\right\}+\Gamma_{\mathrm{WZ}}+\Gamma_{\mathrm{sb}} .
$$

Here $\Gamma_{\mathrm{WZ}}$ is the non-local Wess-Zumino action and $\Gamma_{\mathrm{sb}}$ is the symmetry breaking term. Their explicit form can be found, for instance, in Ref. [12]. In Eq.(11) the left current $L_{\mu}$ is expressed in terms of the chiral field $U$ as $L_{\mu}=U^{\dagger} \partial_{\mu} U$.

Next, the Callan-Klebanov ansatz [9] is introduced

$$
U=\sqrt{U_{\pi}} U_{K} \sqrt{U_{\pi}}
$$

where

$$
U_{K}=\exp \left[i \frac{\sqrt{2}}{f_{K}}\left(\begin{array}{cc}
0 & K \\
K^{\dagger} & 0
\end{array}\right)\right], \quad K=\left(\begin{array}{c}
K^{+} \\
K^{0}
\end{array}\right)
$$


and $U_{\pi}$ is the soliton background field written as a direct extension to $S U(3)$ of the $S U(2)$ field $u_{\pi}$, i.e.,

$$
U_{\pi}=\left(\begin{array}{cc}
u_{\pi} & 0 \\
0 & 1
\end{array}\right)
$$

with $u_{\pi}$ being the conventional hedgehog solution $u_{\pi}=\exp [i \vec{\tau} \cdot \hat{r} F(r)]$.

According to the usual procedure, one expands up to the second order in the kaon field. The Lagrangian density can therefore be rewritten as the sum of a pure $S U(2)$ Lagrangian depending on the chiral field only and an effective Lagrangian describing the interaction between the soliton and the kaon fields. The soliton profile is obtained by minimizing the classical $\mathrm{SU}(2)$ energy while the kaon field satisfies the eigenvalue equation

$$
\left[-\frac{1}{r^{2}} \frac{d}{d r}\left(r^{2} h \frac{d}{d r}\right)+m_{K}^{2}+V_{e f f}^{\Lambda, l}-f \omega_{\Lambda, l}^{2}-2 \lambda \omega_{\Lambda, l}\right] k_{\Lambda, l}(r)=0
$$

where a mode decomposition of the kaon field in terms of the grand spin $\vec{\Lambda}=\vec{L}+\vec{T}(\vec{L}$ represents the angular momentum operator and $\vec{T}$ is the isospin operator) has been used. In Eq.(5) $\omega_{\Lambda, l}$ is the bound state energy for given $(\Lambda, l)$. The radial functions $h, f, \lambda$ and $V_{e f f}^{\Lambda, l}$ are functions of the chiral angle $F(r)$ only. Their explicit forms can be found e.g. in Ref.11.

In this picture strange hyperons arise as bound states of kaons to the soliton. The octet and decuplet hyperons are obtained by populating the lowest kaon bound state which carries the quantum numbers $\Lambda=1 / 2, l=1$. The splittings among hyperons with different spin and/or isospin are given by the rotational corrections, introduced according to the time-dependent rotations:

$$
\begin{aligned}
u_{\pi} & \rightarrow A u_{\pi} A^{\dagger}, \\
K & \rightarrow A K
\end{aligned}
$$

This transformation adds an extra term of order $1 / N_{c}$ to the Lagrangian. The resulting mass formula which takes into account these rotational corrections can be written as

$$
M_{I, J, \mathcal{S}}=M_{\text {sol }}+\omega|\mathcal{S}|+\frac{1}{2 \Theta}\left[c J(J+1)+(1-c) I(I+1)+\frac{c(c-1)}{4}|\mathcal{S}|(|\mathcal{S}|+2)\right] .
$$

Here, $I, J$ and $\mathcal{S}$ are the isospin, spin and strangeness hyperon quantum numbers respectively. $M_{\text {sol }}$ is the soliton mass, $\Theta$ its moment of inertia and $c$ is the hyperfine splitting constant. Their explicit form are given, e.g. in Ref. 13. 
As mentioned above, in this work we are interested in the radiative decays of the decuplet hyperons. Namely, in the processes

$$
\begin{aligned}
& \Sigma^{*} \rightarrow \Lambda \gamma, \\
& \Sigma^{*} \rightarrow \Sigma \gamma, \\
& \Xi^{*} \rightarrow \Xi \gamma .
\end{aligned}
$$

For all these processes both M1 and E2 transitions are allowed. Using the usual multipole expansion of the e.m. field [14, the M1 partial decay width for these processes is given by

$$
\Gamma_{M 1}=18 \alpha q\left|<\hat{M}_{3}(q)>\right|^{2}
$$

where the matrix element is taken between a decuplet hyperon and an octet hyperon, both of them in states with spin projection $J_{3}=+1 / 2$. In Eq.(91), $\alpha=1 / 137$ is the e.m. fine structure constant and $q$ is the photon momentum. The operator $\hat{M}_{3}(q)$ is defined by

$$
\hat{M}_{3}(q)=\frac{1}{2} \epsilon_{3 i j} \int d^{3} r \frac{j_{1}(q r)}{r} r_{i} J_{j}^{e m}
$$

where $j_{1}(q r)$ represents the $l=1$ spherical Bessel function and $J_{j}^{e m}$ are the spatial components of the electromagnetic current.

On the other hand, the E2 partial decay width is given by

$$
\Gamma_{E 2}=\frac{675}{8} \alpha q\left|<\hat{Q}_{33}(q)>\right|^{2}
$$

where the operator $\hat{Q}_{33}(q)$ is given by

$$
\hat{Q}_{33}(q)=\int d^{3} r \frac{j_{2}(q r)}{r^{2}}\left(z^{2}-\frac{r^{2}}{3}\right) \rho^{e m} .
$$

Here $j_{2}$ represents the $l=2$ spherical Bessel function and $\rho^{e m}$ the electric charge density. It should be noticed that in deriving Eq.(11) the Siegert's theorem [14 has been used. For the typical photon momenta and hyperon radii involved in the hyperon radiative decays we have $q r \approx 1$. In this case, the condition $j_{l-1}(q r)>>$ $j_{l+1}(q r)$ necessary for the theorem to be valid, is rather well satisfied for quadrupole transitions. One important advantage of this method is that inconsistencies due to the collective coordinate quantization of the soliton are avoided [7].

In the bound state soliton model the electromagnetic current $\vec{J}^{e m}$ and charge density $\rho^{e m}$ are obtained from the effective action by means of the Noether theorem. 
Given their explicit forms the operators $\hat{M}_{3}(q)$ and $\hat{Q}_{33}(q)$ can be obtained by using the Callan-Klebanov ansatz. After some algebra we get

$$
\hat{M}_{3}(q)=\eta_{s, S}(q) J_{3}^{S}+\eta_{s, K}(q) J_{3}^{K}-2\left(\eta_{v, S}(q)+|\mathcal{S}| \eta_{v, K}(q)\right) R_{33}
$$

where $J^{S}$ the collective angular momentum, $J^{K}$ the bound kaon spin, $R_{i j}=\frac{1}{2} \operatorname{Tr}\left[\tau_{i} A \tau_{j} A^{\dagger}\right]$ and

$$
\begin{aligned}
\eta_{s, S}(q)= & -\frac{1}{3 \pi \Theta} \int d r r j_{1}(q r) \sin ^{2} F F^{\prime} \\
\eta_{v, S}(q)= & \frac{2 \pi}{3} f_{\pi}^{2} \int d r r j_{1}(q r) \sin ^{2} F\left[1+\frac{1}{e^{2} f_{\pi}^{2}}\left(F^{2}+\frac{\sin ^{2} F}{r^{2}}\right)\right] \\
\eta_{s, K}(q)= & c \eta_{s, S}(q)-\frac{2}{3} \int d r r j_{1}(q r)\left\{k^{2} \cos ^{2} \frac{F}{2}\right. \\
& \left.\quad+\frac{1}{4 e^{2} f_{K}^{2}}\left[4 \frac{k^{2}}{r^{2}} \sin ^{2} F \cos ^{2} \frac{F}{2}+k^{2} F^{\prime 2} \cos ^{2} \frac{F}{2}+3 k k^{\prime} F^{\prime} \sin F\right]\right\}, \\
\eta_{v, K}(q)=\frac{1}{6} \int d r r j_{1}(q r)\left\{k^{2} \cos ^{2} \frac{F}{2}\left(1-4 \sin ^{2} \frac{F}{2}\right)\right. & \quad+\frac{1}{4 e^{2} f_{K}^{2}}\left[4 \frac{k^{2}}{r^{2}} \sin ^{2} F \cos ^{2} \frac{F}{2}\left(3-8 \sin ^{2} \frac{F}{2}\right)\right. \\
& \quad+k^{2} F^{\prime 2} \cos ^{2} \frac{F}{2}\left(1-18 \sin ^{2} \frac{F}{2}\right)-2 k^{2} \omega^{2} \sin ^{2} F \\
& \left.\left.+2 k^{\prime 2} \sin ^{2} F+3 k k^{\prime} F^{\prime} \sin ^{2}\left(3-4 \sin ^{2} \frac{F}{2}\right)\right]\right\} \\
+ & \frac{N_{c}}{72} \frac{\omega}{f_{K}^{2} \pi^{2}} \int d r r j_{1}(q r)\left(k^{2} \sin ^{2} F F^{\prime}+k k^{\prime} \sin _{2} F\right)
\end{aligned}
$$

In the previous equations the subscripts $s$ and $v$ denote the isoscalar and isovector parts, and $S$ and $K$ the pure soliton and kaon contributions, respectively.

For $\hat{Q}_{33}(q)$ we obtain

$$
\hat{Q}_{33}(q)=\nu_{v, S}(q)\left[J_{3}^{S} R_{33}+\frac{I_{3}}{3}\right]+\nu_{v, K}(q)\left[J_{3}^{K} R_{33}-\frac{J_{a}^{K} R_{3 a}}{3}\right]
$$

where $I_{3}$ is the z-component of the isospin operator and $\nu_{v, S}(q)$ and $\nu_{v, K}(q)$ are

$$
\begin{aligned}
\nu_{v, S}(q)=\frac{8 \pi f_{\pi}^{2}}{15 \Theta} \int d r r^{2} j_{2}(q r) \sin ^{2} F\left[1+\frac{1}{e^{2} f_{\pi}^{2}}\left(F^{2}+\frac{\sin ^{2} F}{r^{2}}\right)\right], \\
\nu_{v, K}(q)=c \nu_{v, S}+\frac{8}{15} \int d r r^{2} j_{2}(q r)\left\{\omega k^{2} \cos ^{2} \frac{F}{2}\right. \\
+\frac{\omega}{4 e^{2} f_{K}^{2}}\left[k^{2} \cos ^{2} \frac{F}{2}\left(F^{\prime 2}+4 \frac{\sin ^{2} F}{r^{2}}\right)+3 k k^{\prime} F^{\prime} \sin F\right] \\
\left.-\frac{N_{c}}{12 \pi^{2} f_{K}^{2}} \frac{\cos ^{2} \frac{F}{2}}{r^{2}}\left[k^{2} F^{\prime} \cos ^{2} \frac{F}{2}-k k^{\prime} \sin F\right]\right\} .
\end{aligned}
$$


In order to calculate the corresponding matrix elements of the transition operator $\hat{M}_{3}(q)$ and $\hat{Q}_{33}$ we have to evaluate the off-diagonal matrix elements of $J_{3}^{S}, J_{3}^{K}, I_{3}$ $R_{33}, J_{3}^{S} R_{33}, J_{3}^{K} R_{33}$ and $J_{a}^{K} R_{3 a}$ between hyperon wave functions. This is done using standard angular momentum techniques. We obtain for the matrix elements of $\hat{M}_{3}$

$$
\begin{aligned}
<\Lambda\left|\hat{M}_{3}\right| \Sigma_{0}^{*}> & =\frac{2 \sqrt{2}}{3}\left[\eta_{v, S}(q)+\eta_{v, K}(q)\right], \\
<\Sigma_{0}\left|\hat{M}_{3}\right| \Sigma_{0}^{*}> & =\frac{\sqrt{2}}{3}\left[\eta_{s, S}(q)-\eta_{s, K}(q)\right], \\
<\Sigma_{ \pm}\left|\hat{M}_{3}\right| \Sigma_{ \pm}^{*}> & =\frac{\sqrt{2}}{3}\left[\eta_{s, S}(q)-\eta_{s, K}(q) \pm\left(\eta_{v, S}(q)+\eta_{v, K}(q)\right)\right], \\
<\Xi_{\underline{0}}\left|\hat{M}_{3}\right| \Xi_{\underline{0}}^{*}> & =\frac{\sqrt{2}}{3}\left[\eta_{s, S}(q)-\eta_{s, K}(q) \pm \frac{4}{3}\left(\eta_{v, S}(q)+2 \eta_{v, K}(q)\right)\right],
\end{aligned}
$$

while for the those of $\hat{Q}_{33}$

$$
\begin{aligned}
<\Lambda\left|\hat{Q}_{33}(q)\right| \Sigma_{0}^{*}> & =-\frac{\sqrt{2}}{6} \nu_{v, S}(q), \\
<\Sigma_{0}\left|\hat{Q}_{33}(q)\right| \Sigma_{0}^{*}> & =0, \\
<\Sigma_{ \pm}\left|\hat{Q}_{33}(q)\right| \Sigma_{ \pm}^{*}> & =\mp \frac{\sqrt{2}}{6}\left[\nu_{v, S}(q)-\frac{\nu_{v, K}(q)}{6}\right], \\
<\Xi_{\underline{0}}\left|\hat{Q}_{33}(q)\right| \Xi^{*} \underline{0}> & =\mp \frac{4 \sqrt{2}}{27} \nu_{v, K}(q) .
\end{aligned}
$$

It should be noticed that when $q r<<1$ the Bessel functions appearing in the expressions for the $\eta$ 's and $\nu$ 's functions can be replaced by their small argument approximations. In this case we have

$$
\begin{aligned}
& \hat{M}_{3}(q) \rightarrow \frac{q}{3} \mu_{3}, \\
& \hat{Q}_{33}(q) \rightarrow \frac{q^{2}}{15} Q_{33} .
\end{aligned}
$$

Here, $\mu_{3}$ is the static magnetic moment operator and $Q_{33}$ the static electric quadrupole moment operator. It should be kept in mind however that, as mentioned above, $q r \approx 1$ for the transitions we are interested in. In this case, therefore, this "static" approximation is not expected to be very good.

Finally we give the expressions for the E2/M1 ratios. This is defined in terms of the matrix elements of the E2 and M1 transition amplitudes as [7]

$$
\frac{E 2}{M 1}=\frac{1}{3} \frac{<D(1 / 2)\left|M_{2,1}^{E 2}\right| O(-1 / 2)>}{<D(1 / 2)\left|M_{1,1}^{M 1}\right| O(-1 / 2)>} .
$$

Here, $O(-1 / 2)$ represents an octet state with $J_{3}=-1 / 2$ and $D(1 / 2)$ a decuplet state with $J_{3}=+1 / 2$. In terms of the matrix elements of the $\hat{M}_{3}$ and $\hat{Q}_{33}$ operators defined 
above this ratio can be expressed as

$$
\frac{E 2}{M 1}=\frac{5}{4} \frac{<\hat{Q}_{33}>}{<\hat{M}_{3}>} .
$$

Comparing this expression with Eqs.(99,11) we note that the following relation is satisfied

$$
\frac{\Gamma_{E 2}}{\Gamma_{M 1}}=3\left[\frac{E 2}{M 1}\right]^{2} .
$$

In the limit $q r<<1$ Eq.(32) reduces to the expression

$$
\frac{E 2}{M 1} \rightarrow \frac{q}{4} \frac{<Q_{33}>}{<\mu_{3}>}
$$

given in Ref. [7] for the particular case of the $N \gamma \rightarrow \Delta$ process.

We turn now to the numerical calculations. In order to estimate the uncertainties intrinsic in our approach we will use two sets of values for the parameters appearing in the effective action. In SET I we take $m_{\pi}=138 \mathrm{MeV}, f_{\pi}=54 \mathrm{MeV}$ and $e=4.84$. In SET II we consider massless pions and $f_{\pi}=64.5 \mathrm{MeV}$ and $e=5.45$. In both cases we set the ratio $f_{K} / f_{\pi}$ and the kaon mass to their empirical values $f_{K} / f_{\pi}=1.23$ and $m_{K}=495 \mathrm{MeV}$. Results for the total decay widths are given in Table I. In all our calculations we have taken the photon momentum $q$ as the empirical mass difference between initial and final hyperon states 3 , 世. We observe a good agreement between our results and those obtained using the non-relativistic quark model (NRQM) [3, 4, 6] and the bag model (BM) [4] which are also listed in Table I. It should be also noticed that the values obtained by using heavy baryon chiral perturbation theory [5] and quenched lattice QCD [6] span a range which is also consistent with our predictions. This overall agreement between different models contrasts with the situation for the $\Lambda(1405)$ decay widths 11] where the NRQM prediction is much larger than the ones obtained with other models. This can be considered as another indication that contrary to other low-lying hyperons the $\Lambda(1405)$ can not be simply understood as a 3-quark state. Another interesting feature of our results is the strong suppression of the $\Sigma_{-}^{*} \rightarrow \Sigma_{-} \gamma$ and $\Xi_{-}^{*} \rightarrow \Xi_{-} \gamma$ in agreement with the well-known $S U(3) U$-spin selection rule 15. This might be understood by noting that, although in the bound state approach strangeness degrees of freedom are treated rather differently from the isospin ones, at the level of the effective lagrangian the $S U(3)$ breaking is still not so strong. In Table I we have also included the decay width of the only allowed octet-to-octet hyperon transition, namely $\Sigma_{0} \rightarrow \Lambda \gamma$ which is, of course, purely M1. Presently, this is the only radiative hyperon decay for which the empirical decay 
width is accurately known. As we see, in this case the soliton model prediction is reasonable good. It is worthwhile to mention that, in the static limit, the corresponding transition magnetic moment has already been evaluated in Ref. 16.

The predicted $E 2 / M 1$ ratios for the decuplet-to-octet transitions are given in table II. As expected, for the " $U$-spin allowed" transitions we obtain rather small values for the corresponding ratios. On the other hand, for the " $U$-spin forbidden" processes $\Sigma_{-}^{*} \rightarrow \Sigma_{-} \gamma, \Xi_{-}^{*} \rightarrow \Xi_{-} \gamma$ they are quite large. Similar results $₫$ have been reported in Refs. [5, 6] although our values for the forbidden transitions are larger. Since for these transitions both the $M 1$ and $E 2$ amplitudes are quite small, the precise values of the $E 2 / M 1$ become more sensitive to the details of each model and quantitative differences are expected to happen. A particular feature of our predictions is the vanishing of the E2 amplitude (and therefore of the E2/M1 ratio) corresponding to the $\Sigma_{0}^{*} \rightarrow \Sigma_{0} \gamma$ decay. This is a consequence of the spherical symmetry of the isoscalar charge density in the soliton model. As it can be observed in Eq.(18) the operator $\hat{Q}_{33}$ has only isovector components and therefore its matrix elements between states with the same isospin turn out to be proportional to the isospin projection.

In conclusion, we have studied the radiative transitions between decuplet and octet hyperons within the bound state soliton model. We have found that the predictions for the full decay widths are in reasonable agreement with those obtained in quark based models. In particular, the " $U$-spin" selection rule is quite well satisfied. Predictions for the $E 2 / M 1$ ratios have also been made. Corresponding values for the $\Sigma_{-}^{*} \rightarrow \Sigma_{-} \gamma$ and $\Xi_{-}^{*} \rightarrow \Xi_{-} \gamma$ have been found to be quite large. Experimental verification of these predictions as well as those on the e.m. decays of the other excited hyperons will certainly help to improve our understanding on the structure of the hyperons.

The authors wish to thank W. Weise for useful discussions and G.L.Thomas for a private communication on Eq.(17). Most of the work reported here was done while two of them (CLS and NNS) were participating at the INT-95-1 Session on "Chiral Dynamics in Hadrons and Nuclei" at the Institute for Nuclear Theory at the University of Washington, USA. They wish to thank the organizers of the Session for the invitation to participate at it and the Department of Energy, USA for partial financial support during that period. CLS was partially supported by Fundación Antorchas and CG by University of Pavia under the Postdoctoral Fellowship Program.

\footnotetext{
* Our results have an overall sign difference with respect to those quoted in Refs. [5, 6. This is probably due to a difference in the way the E2/M1 ratio is defined. Here, we follow the conventions used in Refs. [7, 14].
} 


\section{References}

[1] R.A. Schumacher, Nucl. Phys. A585 (1995) 63c.

[2] J.S. Russ, Nucl. Phys. A585 (1995) 39c; M.A. Moinester, Proc. of Workshop on Chiral Dynamics, MIT, July 1994, eds. A. Bernstein and B. Holstein (LANL preprint hep-ph/9409463).

[3] J.W. Darewych, M. Horbatsch and R. Koniuk, Phys. Rev. D28 (1983) 1125.

[4] E. Kaxiras, E.J. Moniz and M. Soyeur, Phys. Rev. D32 (1985) 695.

[5] M.N. Butler, M.J. Savage and R.P. Springer, Nucl. Phys. B399 (1993) 69; Phys. Lett. B304 (1993) 353; Phys. Lett. B314 (1993) 122 (E).

[6] D.B. Leinweber, T. Draper and R.M. Woloshyn, Phys. Rev. D48 (1993) 2230.

[7] A. Wirzba and W. Weise, Phys. Lett. B188 (1987) 6; T. Watabe, C.V. Christov and K. Goeke, Phys. Lett. B349 (1995) 197.

[8] R. Davidson, N.C. Mukhopadhyay and R. Wittman, Phys. Rev. Lett. 56 (1986) 804; F. Beck, contribution to the th Conference on Perspectives in Nuclear Physics at Intermediate Energies, Trieste, May 8-12, 1995.

[9] C.G. Callan and I. Klebanov, Nucl. Phys. B262 (1985) 365.

[10] N.N. Scoccola, H. Nadeau, M.A. Nowak and M.Rho, Phys. Lett. B201 (1988) 425; C.G. Callan, K. Hornbostel and I. Klebanov, Phys. Lett. B202 (1988) 269; U. Blom, K. Dannbom and D.O. Riska, Nucl. Phys. A493 (1989) 384.

[11] C.L. Schat, N.N. Scoccola and C. Gobbi, Nucl. Phys. A585 (1995) 627.

[12] D.O. Riska and N.N. Scoccola, Phys. Lett. B265 (1991) 188; M. Rho, D.O. Riska and N.N. Scoccola, Z. Phys. A341 (1992) 343.

[13] Y. Oh, D.-P. Min, M. Rho and N.N. Scoccola, Nucl. Phys. A534 (1991) 493.

[14] J.M. Eisenberg and W. Greiner, Nuclear Theory, Vol.2 (North-Holland, Amsterdam, 1976) p.89.

[15] H.J. Lipkin, Phys. Rev. D7 (1973) 846; H.J. Lipkin and M.A. Moinester, Phys. Lett. B287 (1992) 179.

[16] J. Kunz and P.J. Mulders, Phys. Rev. D41 (1990) 1578. 


\section{Table I}

\begin{tabular}{|c|c|c|c|c|c|}
\hline & \multicolumn{2}{|c|}{ This work } & NRQM & BM & EMP \\
\cline { 2 - 3 } & SET I & SET II & & & \\
\hline$\Sigma_{0} \rightarrow \Lambda \gamma$ & 8 & 7 & 8.5 & 4.6 & $8.9 \pm 0.7$ \\
$\Sigma_{0}^{*} \rightarrow \Lambda \gamma$ & 243 & 170 & 273 & 152 & \\
$\Sigma_{0}^{*} \rightarrow \Sigma_{0} \gamma$ & 19 & 11 & 18 & 15 & \\
$\Sigma_{+}^{*} \rightarrow \Sigma_{+} \gamma$ & 91 & 59 & 110 & & \\
$\Sigma_{-}^{*} \rightarrow \Sigma_{-} \gamma$ & 1 & 1 & 2.5 & & \\
$\Xi_{0}^{*} \rightarrow \Xi_{0} \gamma$ & 148 & 97 & 135 & & \\
$\Xi_{-}^{*} \rightarrow \Xi_{-} \gamma$ & 5 & 5 & 3.2 & & \\
\hline
\end{tabular}

Hyperon radiative decay widths (in $\mathrm{keV}$ ) as calculated in the bound state soliton model. Note that the partial M1 and E2 widths can be obtained from the total widths listed here by using Eq.(33) together with the predictions for E2/M1 listed in Table II. Also listed are the predictions of the non-relativistic quark model (NRQM) [3, 目, 6] and bag model (BM) [四]. The predicted $\Sigma_{0} \rightarrow \Lambda \gamma$ decay width is also included together with the corresponding empirical value.

\section{Table II}

\begin{tabular}{|c|c|c|}
\hline & SET I & SET II \\
\hline$\Sigma_{0}^{*} \rightarrow \Lambda$ & -4.56 & -5.43 \\
$\Sigma_{0}^{*} \rightarrow \Sigma_{0}$ & 0 & 0 \\
$\Sigma_{+}^{*} \rightarrow \Sigma_{+}$ & -4.84 & -7.61 \\
$\Sigma_{-}^{*} \rightarrow \Sigma_{-}$ & -57.7 & -51.1 \\
$\Xi_{0}^{*} \rightarrow \Xi_{0}$ & -3.13 & -4.38 \\
$\Xi_{-}^{*} \rightarrow \Xi_{-}$ & -17.8 & -18.5 \\
\hline
\end{tabular}

Ratios E2/M1 (in \%) as calculated in the bound state soliton model. 